\title{
Prediction accuracy of reservoir break flood simulation model using finite volume method and UAV
}

\author{
Jeongbae Jeon ${ }^{1}$, Won Choi $^{2 *}$ \\ (1. Spatial Information Research Institute, Korea Land and Geospatial Infomatix Corporation, Korea Land and Geospatial Infomatix \\ Corporation, Jeollabuk-do, South Korea; \\ 2. Department of Rural Systems Engineering, Global Smart Farm Convergence Major, Research Institute of Agriculture and Life Sciences, \\ Seoul National University, Seoul 08826, South Korea)
}

\begin{abstract}
Methods for producing high-resolution digital topographic maps using an unmanned aerial vehicle (UAV), and 3D fluid dynamics simulation to estimate the flooded areas caused by a collapsed reservoir, were proposed in this paper. The UAV flight path for photographing damaged areas was divided into two sections considering the drone flight time and overlapping range of the images in the $x$ - and $y$-directions. The metadata taken by the drone were transferred into world coordinates by tracking the key features of the photographs of nearby areas using a 3D rotation matrix. The point cloud data with a 3D space were extracted from the registered images, and a digital surface map (DSM) was produced using a point cloud classification geometric mapping technique. To amend the serious elevation errors caused by natural or artificial obstacles, a kriging interpolation method was used to reproduce the DSM. A transient computational simulation that considers both the complex geometric topology and hydrodynamic energy of flowing water was conducted using FLOW-3D software to deal with an renormalization group (RNG) turbulence model. The flooded areas calculated through visual reading using images taken by the UAV were compared with the $3 \mathrm{D}$ simulation results for verification. The flooded areas estimated through the simulation were approximately $18.3 \%$ larger than those found by visual reading. Turbulent flows were mainly observed in obstacles or curved areas of the stream, and the differences in the water depth could be further increased. However, the villagers confirmed that the flooded areas were much greater than what was seen through the visual reading. Therefore, the combination of UAV surveying and the 3D simulation method based on the RNG turbulence model is recommended to accurately estimate flooded areas, and it will support an administrative policy aimed at minimizing the economic costs of damage caused by future reservoir collapses.
\end{abstract}

Keywords: reservoir failure, flooded area, finite volume method, unmanned aerial vehicle, photogrammetric survey, digital surface model

DOI: $10.25165 /$ j.ijabe.20201306.4909

Citation: Jeon J, Choi W. Prediction accuracy of reservoir break flood simulation model using finite volume method and UAV. Int J Agric \& Biol Eng, 2020; 13(6): 7-15.

\section{Introduction}

There are 17569 agricultural reservoirs distributed throughout South Korea, which play an important role in supplying approximately $54 \%$ of the required agricultural water, although their storage capacities are relatively small. However, most of these reservoirs have been around for more than 50 years, and approximately $68 \%$ of them were constructed before $1945^{[1]}$. Since 2000, large-scale natural disasters, such as typhoons Maemi and Rusa, have caused massive flood damage accompanied by heavy or localized torrential downpours that exceeded the design capacities of the reservoirs, destroying several of them ${ }^{[2,3]}$. For example, the Chunjeong and Daesa reservoirs in Boseong and Goheung counties in Jeollanam-do province, respectively, were

Received date: 2019-03-10 Accepted date: 2020-09-22

Biographies: Jeongbae Jeon, $\mathrm{PhD}$, research interests: safety and disaster prevention engineering, Email: jbjeon@1x.or.kr.

*Corresponding author: Won Choi, PhD, Associate Professor, research interests: numerical analysis and simulation. Department of Rural Systems Engineering, Global Smart Farm Convergence Major, Research Institute of Agriculture and Life Sciences, College of Agriculture and Life Sciences, Seoul National University, 1 Gwanak-ro, Gwanak-gu, Seoul 08826, South Korea. Tel: +82-2-880-4580, Email: fembem@snu.ac.kr. destroyed in 2007 owing to embankment overflows caused by localized torrential heavy rains reaching more than $100 \mathrm{~mm}$ per hour $^{[4]}$. In August 2012, nine reservoirs in Gochang-gun county in Jeollabuk-do collapsed or were destroyed by typhoon Bolaven; of these, embankment collapses included the losses of spillways in the Jukrim and Yulji reservoirs ${ }^{[5]}$. In July 2013, a heavy rain of more than $300 \mathrm{~mm}$ in Gochang-gun County in Jeollabuk-do destroyed the retaining wall of the Josan reservoir, which was under construction to increase the height of the embankment, and resulted in the evacuation of approximately 2000 residents ${ }^{[6]}$.

The structural safety of reservoirs in South Korea is rated as A, $\mathrm{B}, \mathrm{C}$, or $\mathrm{D}$ based on the material reliability and boundary condition according to age and a change in geology, respectively. According to a national reservoir management status report published by the Korea Infrastructure Safety \& Technology Co. in 2013, 1545 out of 3347 reservoirs managed by the Korea Rural Community Corporation (KRC) have been inspected for their structural safety, and $1474(95 \%)$ were rated at the lowest levels (C or D class). Therefore, the report indicates that almost all the reservoirs are in danger of a collapse.

Because human and material damages to downstream areas are caused by reservoir failures, research on disaster preparedness systems to prevent flood damage is being actively conducted in 
South Korea. Since 2004, the National Emergency Management Agency has prepared emergency action plans for agricultural reservoirs and has proposed flood maps and reinforcement methods for existing reservoirs considering the risk of disaster ${ }^{[7]}$. Kim et al. ${ }^{[8]}$ estimated the hazardous flood zones following excessive rainfall in the Munsan River Basin in Gyeonggi-do province, using the 1D hydraulic Hydrologic Engineering Center - River Analysis System (HEC-RAS) model. Cho et al. ${ }^{[9]}$ produced flood hazard maps in the Geumho River Basin, a tributary of the Nakdong River, using the 2D hydraulic model, FLUMEN.

HEC-RAS 1D and $2 \mathrm{D}^{[10-14]}$ are the representatives of existing commercial or open-source programs that were originally based on both a one-dimensional energy equation and a steady state flow, meaning that there is an indirect method that does not reflect the hydraulic effect caused by the cross-section shape, stream bends, or other factors. Energy losses related to friction and contraction/expansion can be evaluated based on the assumption of an engineer's subjective opinion ${ }^{[15]}$. In addition, the HEC-RAS simply assumes that if the ground levels on the riverside, the shape of which is not feasible to contain water, are lower than the flood water level of the river in the protected lowland, the protected lowland will become flooded ${ }^{[16]}$. However, because actual flood waves have the characteristics of fluids such as potential and kinetic energies and turbulence, the exact downstream damage owing to reservoir failures should be estimated through an unsteady $3 \mathrm{D}$ hydraulic dynamics simulation depending on time ${ }^{[17,18]}$.

To execute 1D or 2D numerical simulations analyzing downstream areas damaged by reservoir breaks, initial input data such as the amount of flood discharge and topological information are required. The amount of flood discharge is generally subjective data calculated by an engineer ${ }^{[19-21]}$. The topological data can be obtained from the contours in digital topographic maps or digital elevation models (DEMs) using satellite images. However, there are risks involved in inaccurately representing the boundary heights of the paddies, dry fields, and roads, among other areas, as well as in using DEMs with a low spatial resolution. Although the cost of satellite images is low, the accuracy of the results is reduced ${ }^{[22,23]}$. For example, Landsat images with a spatial resolution of $30 \mathrm{~mm}$ are provided free of charge, but it is difficult to generate accurate cross-sectional views using such images. As an alternative, actual field surveys are often needed to obtain high-resolution cross-sectional views. However, because river cross-sections are surveyed only for each station, sectional continuity is not guaranteed.

Airplane images provide accurate geographical and cross-sectional data for certain areas within a short period of time, particularly when using unmanned aircraft flyable at low altitudes $^{[24,25]}$. Because unmanned aerial vehicles (UAVs) also have the advantage of obtaining spatial information easily in disaster areas where immediate access is difficult, its cost is relatively low in comparison to detailed wide-field surveys ${ }^{[26]}$. In the field of agricultural disaster prevention, these features provide vital information for identifying and addressing problems that are difficult to obtain using ground research, satellites, and aviation imagery ${ }^{[27]}$.

In determining the extent of flood damage, such as after the recent and frequent reservoir losses in South Korea, the UAV offers convenience and ease of accessibility to provide geographical information quickly and accurately, which is not possible with traditional approaches. Thus, it can solve problems requiring a significant amount of money, procedures, and time to acquire aerial images, making it possible to assess the situation before a disaster, understand what is required to change the situation, and identify the current progress of the recovery. The ability of a UAV to function remotely is an extremely useful feature in disaster management, enabling it to acquire information in areas that are inaccessible because of certain reasons, e.g., a reservoir accident ${ }^{[28]}$.

Central or local governments have tallied the social and economic damage incurred by downstream floods after reservoir failures, and every year, a considerable monetary budget is spent to compensate for human fatalities in concentrated residential areas. Thus, to set aside possible financial support of the government, it is necessary to accurately estimate the potential downstream damages associated with any reservoir failure before they occur. Therefore, in this study, a UAV was used to create a precise topographical map that was useful for monitoring the areas of damage by the actual loss of a reservoir. The inundation areas where the disaster occurred were calculated using a 3D computational fluid dynamics simulation. This method can thus be used to predict the probable flood damage associated with future reservoir losses.

\section{Materials and methods}

\subsection{Targeted area}

For a numerical simulation of flooded areas caused by the actual collapse of a reservoir dam body, the Goeyeon reservoir was selected, which was built in 1945. It is located in the Goeyeon-dong district, Yeongcheon City, in Gyeongsangbuk-do province, South Korea (Figure 1). The length and height of the reservoir embankment are 160 and $5.5 \mathrm{~m}$, respectively, and the capacity of the reservoir is $61400 \mathrm{~m}^{3}$. A concentrated torrential rainfall event was recorded by the Korea Meteorological Administration (KMA) in the area from August 17 to 21, 2014; the total rainfall was $290 \mathrm{~mm}$, and approximately $65 \mathrm{~mm}$ fell during a 3-h period prior to the failure of the embankment and spillway of the reservoir. Approximately 500 residents from nearby areas were evacuated, and most agricultural belts, including vineyards, cornfields, and lower-level roads, were washed away by the flood.

The Rural Agricultural Water Resource Information System (RAWRIS) of the KRC in South Korea provides basic data on the irrigation facilities associated with all rural waters. Information regarding the Goeyeon reservoir is shown in Table 1.

\subsection{Digital surface model (DSM)}

Although precise topographic mapping is required to analyze river hydraulics in detail, doing so is time- and labor-intensive as well as expensive. As an alternative to precise surveying, an unmanned aerial photogrammetric survey method was chosen for this research because a UAV can accurately survey an area of approximately $3 \mathrm{~km}^{2}$ in 4 to $5 \mathrm{~h}$. The day after the Goeyeon reservoir failure, a UAV was also used to determine the stage-storage curve of the reservoir.

An eight-rotor UAV (S1000; DJI, Taipei, Taiwan) with a size of $1300 \mathrm{~mm} \times 1300 \mathrm{~mm} \times 140 \mathrm{~mm}$ and a weight of $3.5 \mathrm{~kg}$ was selected, and a NEX-5T camera (Sony, Tokyo, Japan), which is compact, mirrorless, and has interchangeable lenses, was used for photographing the area. The drone flight path for photographing the region was divided into two sections to contain all areas damaged by the failure of the reservoir body, as shown in Figure 2 . 
The overlapping range of the images in both the $x$ - and $y$-directions was empirically set to $60 \%$, and the drone took 338 digital orthoimages (153 and 185 orthoimages in each section).

For each photograph, the latitude $(x)$, longitude $(y)$, and altitude $(z)$, and the pitch $(\omega)$, roll $(\psi)$, and yaw $(\chi)$ metadata of the drone were automatically saved into a $\log$ file. Each set of metadata was transferred into world coordinates and tracked using the key points of the photographs of nearby areas by applying a 3D rotation matrix ${ }^{[29]}$. The point cloud data with a $3 \mathrm{D}$ space were extracted from the registered images, and a DSM was produced in the form of a digital map using PhotoScan software (Ver. 1.3, Agisoft; St. Petersburg, Russia) ${ }^{[30]}$.

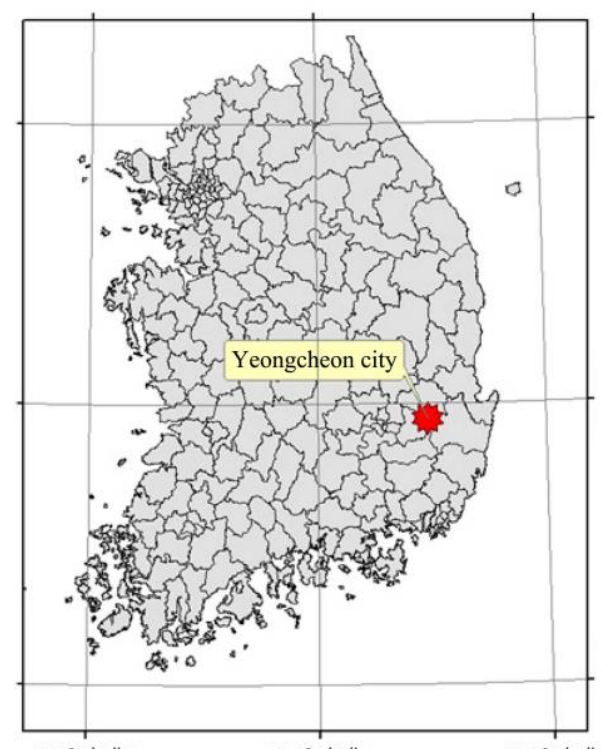

$126^{\circ} 0^{\prime} 0^{\prime \prime} \mathrm{E}$

$128^{\circ} 0^{\prime} 0^{\prime \prime} \mathrm{E}$

$130^{\circ} 0^{\prime} 0^{\prime \prime} \mathrm{E}$

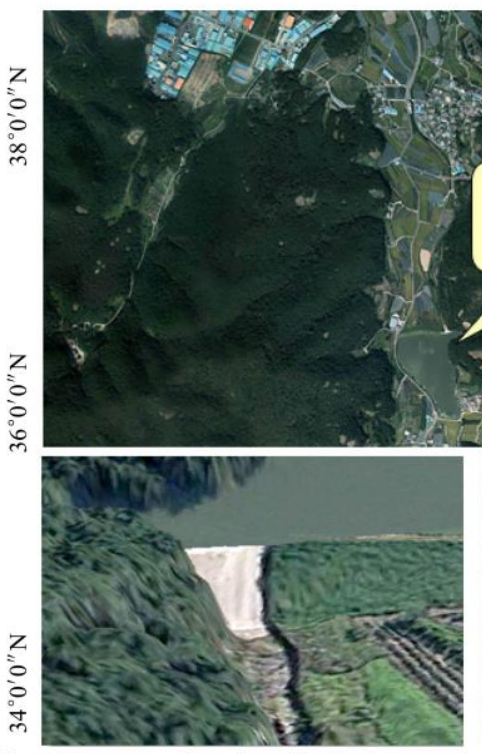

Before the collapse
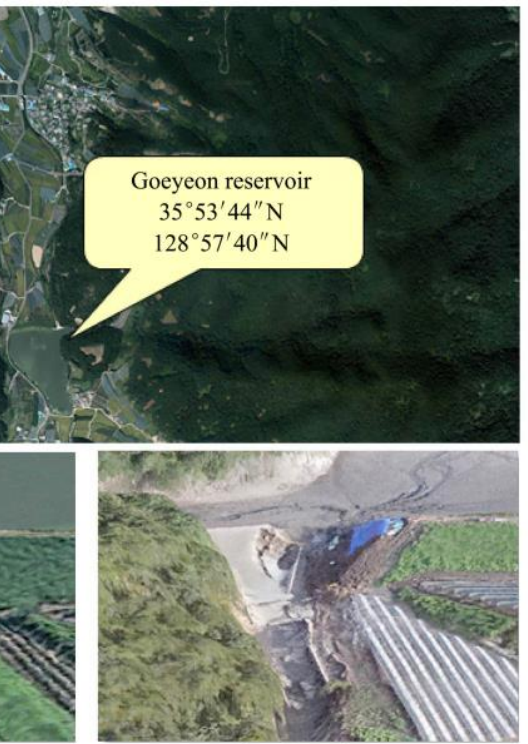

After the collapse

Figure 1 Geodetic location of Goeyeon reservoir

Table 1 RAWRIS data on Goeyeon reservoir

\begin{tabular}{|c|c|c|c|c|c|c|c|}
\hline \multicolumn{2}{|c|}{ Basin } & \multicolumn{4}{|c|}{ Embankment (reservoir body) } & \multicolumn{2}{|c|}{ Spillway } \\
\hline Watershed area $/ \mathrm{hm}^{2}$ & Beneficial area $/ \mathrm{hm}^{2}$ & Length/m & Height $/ \mathrm{m}$ & Upstream slope & Downstream slope & Length/m & Height $/ \mathrm{m}$ \\
\hline 125 & 28 & 160 & 5.5 & $1: 2$ & $1: 2$ & 15 & 2.4 \\
\hline
\end{tabular}

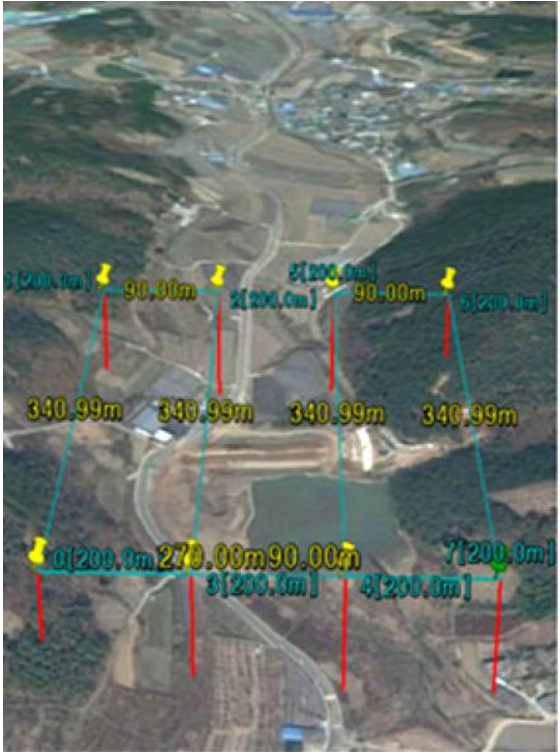

Figure 2 Drone flight paths set up for photogrammetric survey

\subsection{Flood hazard map}

To better predict flood hazard areas, RGB photographic images with a high resolution are needed. This research used RGB images with a spatial resolution of $4 \mathrm{~cm} \times 4 \mathrm{~cm}$ per cell, which can distinguish the outlines of car-sized objects. By comparison, RGB images with a resolution of $20 \mathrm{~cm} \times 20 \mathrm{~cm}$ per cell only distinguish large objects such as houses, roads, and farmland, as shown in Figure 3.

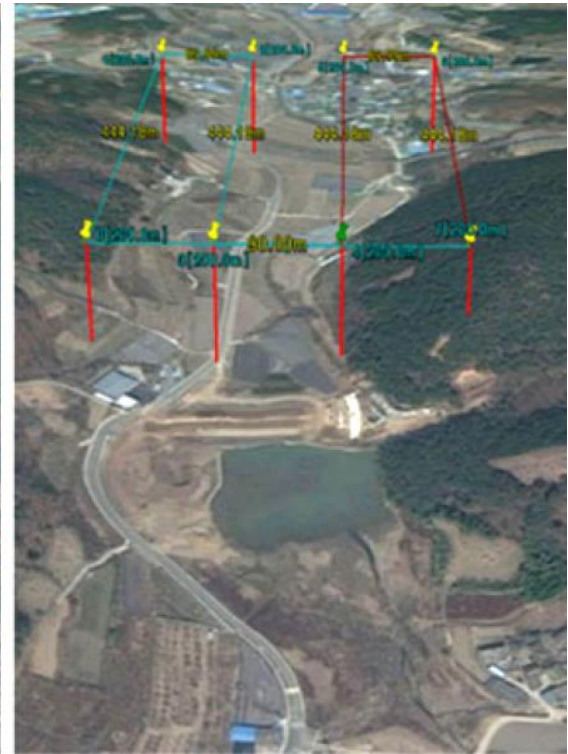

Cross-sections per meter were also generated by the DSM, for a distance of $600 \mathrm{~m}$ downstream from the reservoir. To compare these cross-sections with numerical results for verification, the inundation trace areas per cross-section were marked by the visual reading method shown in Figure 4. A flood hazard map of the downstream areas was finally produced based on the DSM generated from the images captured by the UAV. 

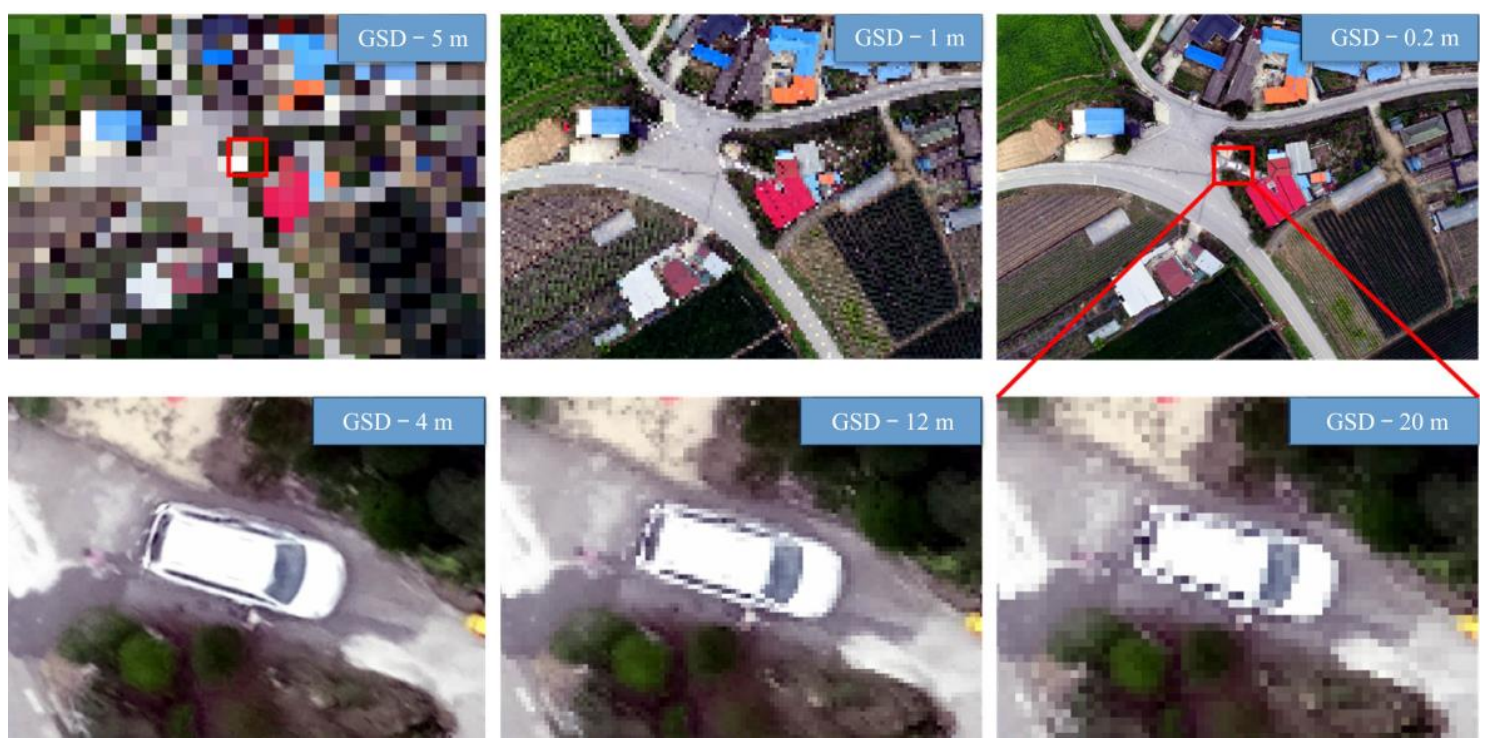

Figure 3 Accuracy of visual assessment, tracking with spatial resolution
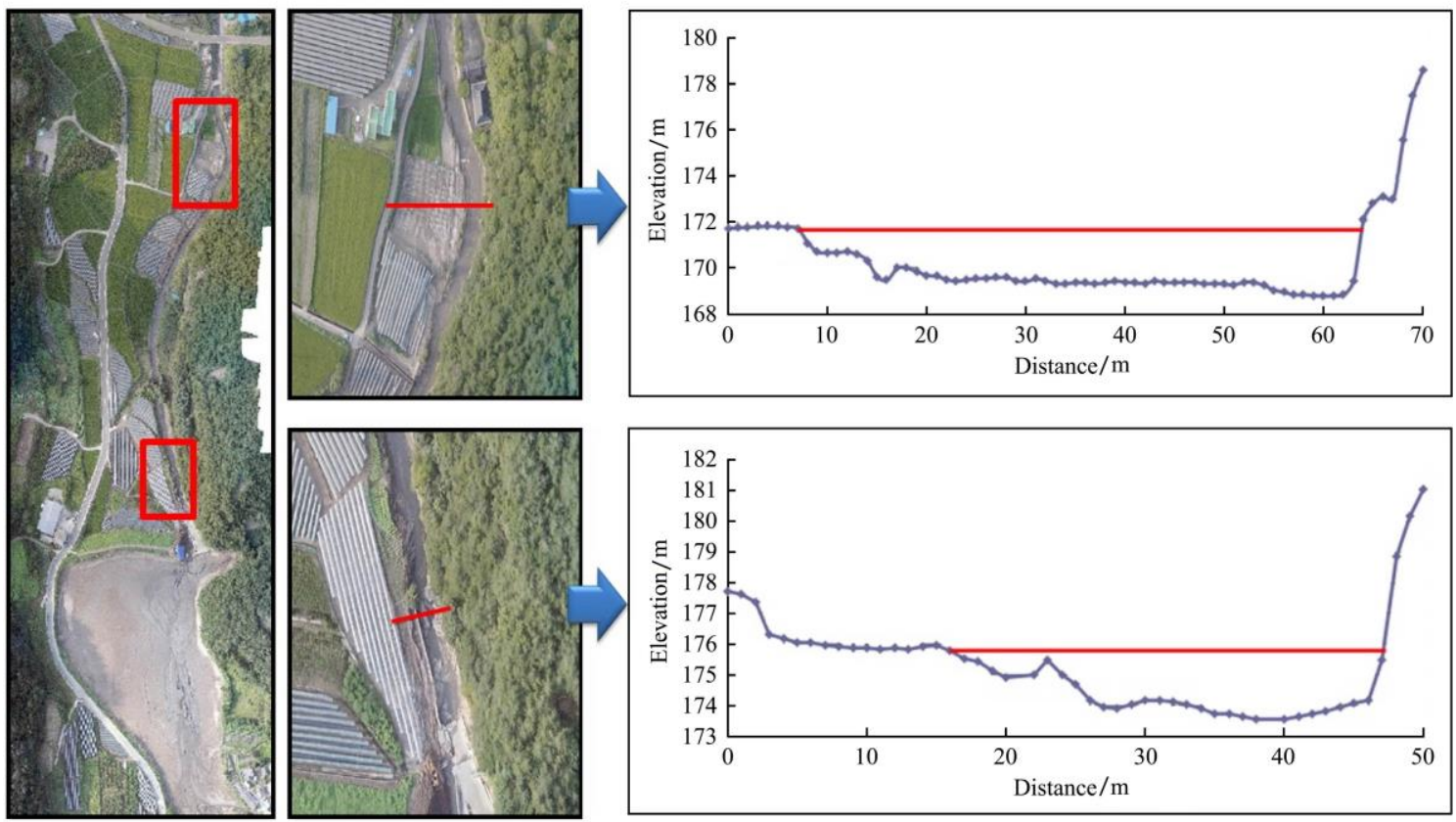

Figure 4 Cross-section drawings and inundation trace areas

\section{Numerical simulation}

To date, many researchers have used various Hydrologic Engineering Center - Hydrologic Modeling System (HEC-HMS) and Dam Break (DAMBRK) models to predict areas of inundation by assuming possible scenarios related to reservoir failures. However, such models fundamentally contain large error ranges because their numerical simulations are based on 1D or 2D hydrodynamic models using interpolated cross-sections along the downstream river. This is why $3 \mathrm{D}$ computational fluid dynamics must be used to reflect both the continuity of the topography and hydrodynamic energy of the flowing water. The commercial software FLOW-3D (Ver. 10.1; Flow Science, Inc., Santa Fe, NM, USA), which is a computational and numerical model, was introduced to simulate the transient flow of an incompressible viscous fluid.

FLOW-3D is based on a finite volume method and is an accurate computational fluid dynamics (CFD) software for dealing with transient and free-surface problems of flowing water. The finite volume method is a numerical method used to solve conservation equations in the form of partial differential equations derived using the divergence theorem. In FLOW-3D, free surfaces of flowing water are calculated through the volume of fluid (VOF) technique, which is a special advection method used to determine the interfaces between water and air in one cell without using a fine mesh. FLOW-3D also employs an innovative meshing method, i.e., a structured fractional area-volume obstacle representation (FAVOR), to eliminate possible numerical flow losses by smoothly blocking the fractional portions of grid cell faces and volumes. In the Cartesian grid system, FAVOR dramatically improves the problem by embedding the geometry directly into the mesh, allowing for rapid parametric adjustments without labor-intensive re-meshing. Over the past few years, many studies have applied numerical simulations of fluid dynamics to understand the spillway effects and dam breaks ${ }^{[17,18,31-34]}$.

\subsection{Governing equations}

In the absence of sources and sinks of fluid water, the conservation equations for incompressible and transient states are divided into mass (continuity) and momentum (Cauchy) equations as follows: 
For the continuity,

$$
\frac{\partial}{\partial x_{i}}\left(u_{i} A_{i}\right)=0
$$

For the momentum,

$$
\frac{\partial u_{i}}{\partial t}+\frac{1}{V_{F}}\left(u_{j} A_{j} \frac{\partial u_{i}}{\partial x_{j}}\right)=-\frac{1}{\rho} \frac{\partial P}{\partial x_{i}}+g_{i}+f_{i}
$$

where, $u$ is the velocity required to move through the face of a cell, $\mathrm{m} / \mathrm{s} ; A$ is the face area of the cell, $\mathrm{m}^{2} ; t$ is the flow time, $\mathrm{s} ; V_{F}$ is the fluid volume fraction of the cell; $\rho$ is the density of the fluid, $\mathrm{kg} / \mathrm{m}^{3}$; $P$ is the pressure of the cell, $\mathrm{Pa} ; g$ is the body acceleration of the cell, $9.8 \mathrm{~m} / \mathrm{s}^{2} ; f$ is the viscous acceleration of the cell, $\mathrm{m}^{2} / \mathrm{s}^{2}$; and $x_{i}$ is the axis in the subscript direction ( $i$ ) of the 3D Cartesian coordinate system.

The viscous acceleration related to dynamic viscosity $(\mu, \mathrm{Pa} \cdot \mathrm{s})$ is expressed as follows:

$$
\rho V_{F} f_{i}=w s x_{i}-\frac{\partial}{\partial x_{j}}\left(A_{j} \tau_{i j}\right)
$$

where, ws $x$ is the wall shear stress, $\mathrm{Pa} ; \tau$ is the shear stress, Pa. $\tau_{i i}=2 \mu\left\{\frac{\partial u_{i}}{\partial x_{i}}-\frac{1}{3}\left(\frac{\partial u_{j}}{\partial x_{j}}\right)\right\} \quad, \quad \tau_{i j}=-\mu\left\{\frac{\partial u_{j}}{\partial x_{i}}+\frac{\partial u_{i}}{\partial x_{j}}\right\} \quad, \quad$ and $(i, j)=(1,2),(1,3), \quad(2,3)$.

In this model, the wall shear stresses are set to zero by assuming that the tangential velocities on the walls are zero. The VOF function $(F)$ used to define the ratio of fluid to the unit volume was applied to track the free surface of the fluid. The VOF transportation equation is defined as follows:

$$
\frac{\partial F}{\partial t}+\frac{1}{V_{F}}\left\{\frac{\partial}{\partial x}\left(F A_{i} u_{i}\right)\right\}=0
$$

The RNG turbulence model with improved calculations for areas of high-pressure gradients at the walls generally has wide applications, with coefficients determined through rigorous statistical analysis. In particular, it is able to describe flows with strong shear regions more accurately and rapidly than the standard $k-\varepsilon$ model. The transport model was applied for this study, and its equations related to the turbulent kinetic energy $\left(k, \mathrm{~m}^{2} / \mathrm{s}^{2}\right)$ and turbulent kinetic energy dissipation rate $\left(\varepsilon, \mathrm{m}^{2} / \mathrm{s}^{2}\right)$ are shown below.

$$
\begin{gathered}
\frac{\partial k}{\partial t}+\frac{1}{V_{F}}\left\{u_{i} A_{i} \frac{\partial k}{\partial x_{i}}\right\}=P+\operatorname{Diff}_{k}-\varepsilon \\
\frac{\partial \varepsilon}{\partial t}+\frac{1}{V_{F}}\left\{u_{i} A_{i} \frac{\partial \varepsilon}{\partial x_{i}}\right\}=C_{1} \frac{\varepsilon}{k} P+\operatorname{Diff}_{\varepsilon}-C_{2} \frac{\varepsilon^{2}}{k}
\end{gathered}
$$

where, $P$ is the turbulent kinetic energy production, $\mathrm{m}^{2} / \mathrm{s}^{2} ;$ Diff $f_{k}$ is the diffusion of $k, \mathrm{~m}^{2} / \mathrm{s}^{2} ;$ Diff $\varepsilon_{\varepsilon}$ is the diffusion of $\varepsilon, \mathrm{m}^{2} / \mathrm{s}^{3}$, and $C_{1}$ and $C_{2}$ are the dimensionless user-adjustable parameters.

In this RNG turbulence model, the values 1.42 and 1.68 were used for $C_{1}$ and $C_{2}$, respectively. In all turbulence transport equations, the kinematic turbulent viscosities are computed as follows:

$$
v=C N U \frac{k^{2}}{\varepsilon}
$$

where, $v$ and $C N U$ are the turbulent kinetic viscosity $\left(\mathrm{m}^{2} / \mathrm{s}\right)$ and constant parameter, respectively. In this model, the value of $C N U$ is set to 0.085 .

\subsection{Material properties and simulation conditions}

For a 3D numerical analysis, the data were constructed using the following processes. First, PhotoScan (Ver. 1.3; Agisoft,
Petersburg, Russia) was used to produce a DSM Raster file from the photographic images acquired from the drone by applying the Structure from Motion (SfM) method. The few parts of the DSM data covered by obstacles in the water flow were manually revised using the Kriging method of ArcGIS software (Ver. 10.0; Esri, Redlands, CA, USA), and the modified DSM was converted into STereoLithography (STL) using the DEMto3D, an extension of QGIS software (Ver. 3.4.3, open-source cross-platform desktop GIS application). The 3D geometry was imported into AutoCAD software (Ver. 2010; Autodesk, Inc., San Rafael, CA, USA), and the complicated geometry was empirically divided into several parts to optimize the memory usage. The FLOW-3D executed the numerical simulation based on the geometry, and the outcome results (the point value data) obtained from the simulation were finally used for the visualization using the 'point to Raster' function of ArcGIS. The I/O data were generated using PhotoScan, ArcGIS, QGIS, and AutoCAD programs at each stage, and the data implementation process is as shown in Figure 5. Because the resolution of the drone images was set to $4 \mathrm{~cm}$ initially, the resolutions during this entire process were kept at $4 \mathrm{~cm}$ to minimize data loss.

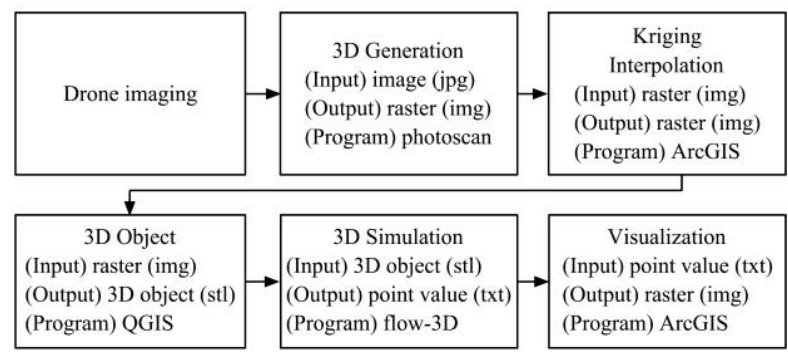

Figure 5 Input and output following data process stage

All parts from which the fluids flowed out of the geometries, as well as parts from which fluids did not flow, were imported into the FLOW-3D software for use as the simulation solid domain. For this site, because the streams have few curves, even two simple rectangular geometries are sufficient for the simulation. However, if the streams are heavily curved, several sizes of geometries must be adjusted. Because the spatial resolution of the original DSM was $4 \mathrm{~cm}$, the size of the mesh blocks was set to less than $4 \mathrm{~cm}$ in order to increase the simulation accuracy. To save computational time, the mesh number and mesh block size of each domain were empirically determined, as shown in Figure 6. No-slip conditions on the boundaries were applied to the ground. The material properties and boundary conditions for the simulation are listed in Table 2.

A generalized minimum residual (GMRES) algorithm implemented in the software was activated to solve the pressures and velocity ${ }^{[35-37]}$. A generalized conjugate gradient (GCG) algorithm was also selected to compute the viscous terms in the GMRES solver. To increase the simulation accuracy, the convergence tolerance and maximum number of iterations per time step were set to 0.001 and 50 , respectively. The time step and total analysis time were set to $1 \mathrm{~s}$ and $60 \mathrm{~s}$, respectively. The dynamic fluid flows that interact with the solid geometries were finally simulated using the FLOW-3D software. It took approximately $242 \mathrm{~h}$ to accomplish the simulation using a server level single computer (Intel ${ }^{\circledR}$ Xeon $^{\circledR}$ CPU X5690@4.00 GHz (two processors), 48 GB of G.SKILL RAM @1600 MHz). When the dam broke, the water exposed to the space newly opened from the reservoir began to flow because of the stagnation pressure due to gravity. 


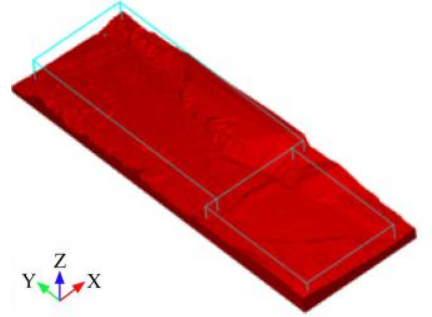

a.

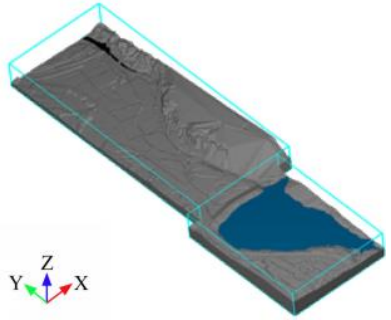

b.
Figure 6 Three-dimensional geometry (a) and water space rendered in FAVOR view (b)

Table 2 Material properties and simulation conditions

\begin{tabular}{lcc}
\hline \multicolumn{1}{c}{ Variable } & Value & Reference \\
\hline Fluid density $/ \mathrm{kg} \cdot \mathrm{m}^{-3}$ & 1000 & $293.15 \mathrm{~K}\left(20^{\circ} \mathrm{C}\right)$ \\
Fluid viscosity $/ \mathrm{Pa} \cdot \mathrm{s}$ & 0.001 & Dynamic viscosity \\
Gravity $/ \mathrm{m} \cdot \mathrm{s}^{-2}$ & 9.8 & \\
Boundary condition & No-slip & \\
\hline
\end{tabular}

\section{Results and discussion}

\subsection{DSM analysis}

The gray-scale image of the DSM was generated from high-resolution photographs taken by the UAV (Figure 7). As per the geographical statistical analysis results of the DSM, the top elevation of the embankment, the lowest elevations of the reservoir, and the downstream river were $183 \mathrm{~m}, 177 \mathrm{~m}$, and $166 \mathrm{~m}$, respectively. It was observed from the elevation image that the river beginning from the embankment of the reservoir flows to the right side, and the farm and forest areas are located on the left and right sides of the main stream of the river, respectively. However because some of the surfaces shown on the map are covered by obstacles such as bridges and submerged weirs, an appropriate method for correcting the elevation errors that create serious problems in fluid dynamics simulations is required. In this study, the Kriging interpolation method, which topologically calibrates noncontinuous areas through a Gaussian process, was used to modify the elevation faults of the DSM generated from point cloud data (Figure 8). Three-dimensional images produced from aerial photographs are based on a surface model. Because this may result in some differences compared to the actual terrain, the obstacle areas such as weirs, bridges, and trees were removed, and a spatial interpolation method was carried out in this study. There are various representative methods of spatial interpolation, including $k$-nearest neighbor, inverse distance weight, and Kriging. The inverse distance weight interpolation is a method for determining the value of a new cell using weights that are joined linearly from nearby points because the closer the distance, the higher the weight applied. This is mainly used in the field for preventing geochemical disasters. By contract, in the field of GIS, the Kriging method is the best interpolation technique in which the value around the point to be interpolated is weighted, and the necessary value is quantitatively evaluated by specifying the spatial patterns. The Kriging method is also a statistical method for predicting attribute values at points of interest through a linear combination of known data. The estimated values reflect not only the distance from the actual measurement but also the correlation relationship between the values adjacent to the surroundings. However, it has a disadvantage in that a new weight in each time step is calculated for a new point, thus requiring a large number of calculations. The Kriging method is the best technique used in the geostatistics field, and was therefore applied in this study ${ }^{[38-41]}$.

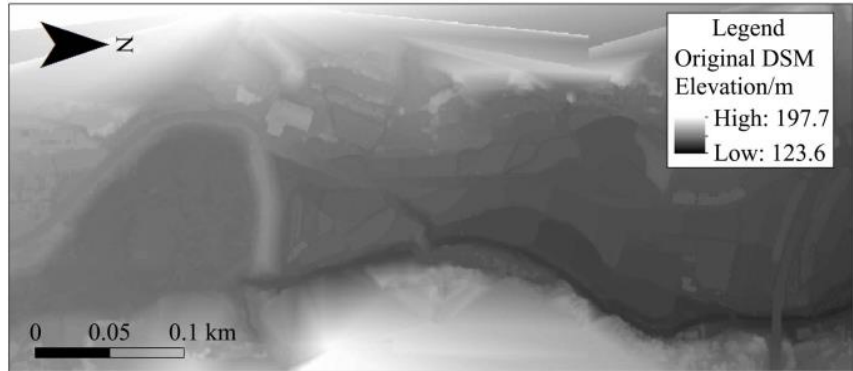

Figure 7 Gray-scale image of DSM generated from point cloud data

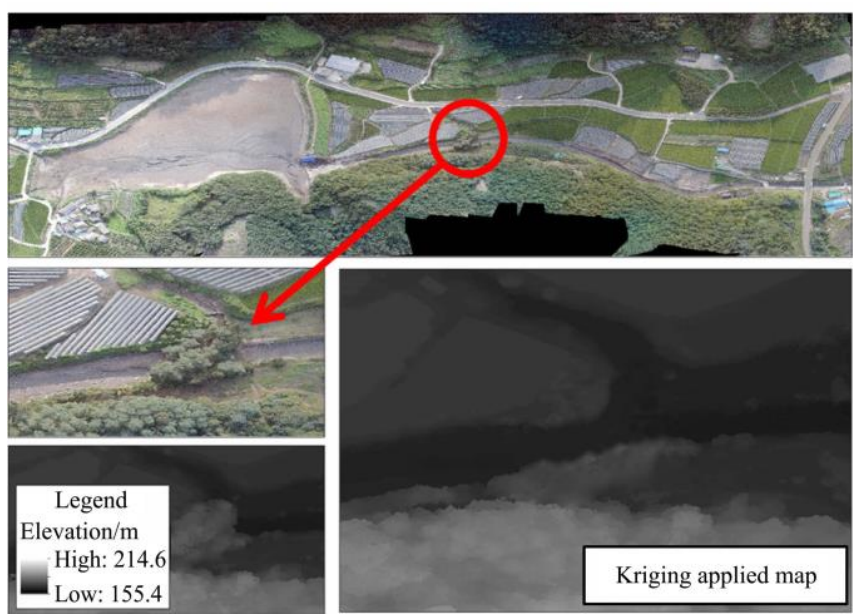

Figure 8 DSM image reproduced by Kriging interpolation method

\subsection{Flooded areas}

Based on the finalized DSM, the flooded areas downstream were estimated to be $18514 \mathrm{~m}^{2}$ by a visual reading and $22211 \mathrm{~m}^{2}$ by a 3D computer simulation method. The areal difference was approximately $18.3 \%$ (Table 3 and Figure 9). The depths of the flooded areas are also shown in Table 3. When the ranges of the flooded depths were below $100 \mathrm{~cm}$ and over $500 \mathrm{~cm}$, the areal differences between the visual reading and computer simulation were relatively smaller than those in the other areas. This is because the 3D simulation method was able to calculate the flowing dynamic energies in the $x$-, $y$-, and $z$-directions to accurately reflect the amount of water overflowing the river when the flow velocities were fast, whereas the visual reading method considered only the current state shown from the photos taken by the drone camera. The visual reading was calculated based on the height of the point at which the flood depth was predicted. Therefore, where the depth of the flooding was so low that it is impossible to predict the damage to crops or the movement of soil, the flooding area may not be identified through a visual reading. However, in the case of a 3D numerical simulation, to deal with the dynamic energy of the fluid, even a low depth of flooding can be analyzed. When there were obstacles, such as weirs in the stream or when the streams were curved, the water levels of the simulation were also rated higher than the visual reading. In addition, flow patterns similar to a laminar flow were mainly found in areas with relatively extremely low or high water levels, and the differences with a visual reading were reduced. By contrast, the turbulence patterns were found to be relative in the middle part of the water depth, and the differences were further increased. It was clear from the results that the visual reading was underestimated. This was also confirmed through on-site visits in which the actual flood depth reported by farmers living in the village at that time was wider than the visual reading. 


\section{Table 3 Flooded areas categorized based on flooded depth}

\begin{tabular}{cccc}
\hline Flooded depth/cm & Visual reading $/ \mathrm{m}^{2}$ & 3D simulation $/ \mathrm{m}^{2}$ & Error ratio/\% \\
\hline Under 50 & 2482 & 2250 & 9.3 \\
$50-100$ & 5062 & 4872 & 3.8 \\
$100-200$ & 4178 & 6504 & 55.7 \\
$200-300$ & 3309 & 3949 & 19.3 \\
$300-400$ & 2352 & 3518 & 49.6 \\
$400-500$ & 653 & 990 & 51.6 \\
Over 500 & 118 & 128 & 8.5 \\
Summation & 18154 & 22211 & 22.3 \\
Difference & & 4057 & \\
\hline
\end{tabular}

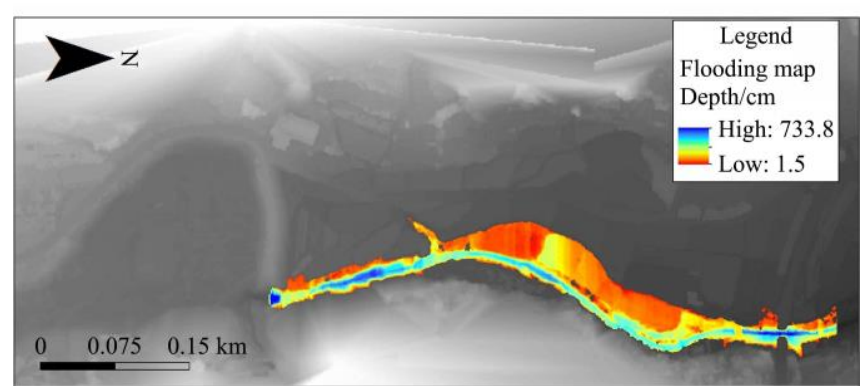

a.

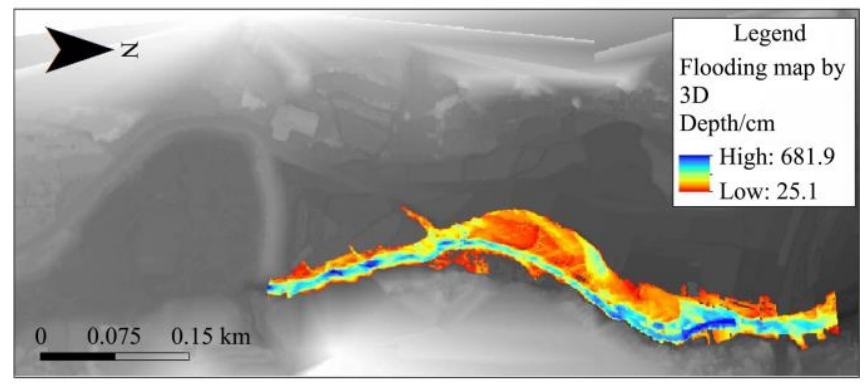

b.

Figure 9 Flooded areas estimated by visual reading (a) and computer simulation methods (b)

The differences in elevation used to subtract the visual readings from the $3 \mathrm{D}$ computer simulation are shown as a pixel $(1 \mathrm{~m} \times 1 \mathrm{~m})$ in Figure 10. The greatest difference was $-381 \mathrm{~cm}$ in one particular small area; in most of the region $\left(16349 \mathrm{~m}^{2}\right)$, the water levels from the 3D computer simulation were slightly higher than those estimated by a visual reading because the level differences were only slightly negative. By contrast, a total flooded area of only $1588 \mathrm{~m}^{2}$ showed elevation differences of more than $200 \mathrm{~cm}$. In Figure 10, area A1 was linearly corrected to a certain height using the Kriging method, which might have reflected the significant differences in water levels. In area A2, the flow velocity was accelerated along the curved stream between $\mathrm{A} 1$ and $\mathrm{A} 2$, and a 3D computer analysis found that the water levels were higher owing to the dynamic energy. A small concrete weir structure was located within the A3 region, and thus some of the flowing water accumulated there, giving it a higher level than the surrounding terrain as compared with the visual reading method. This is evidence that hydraulic structures located at the bottom of a stream could induce a larger difference in the depth of the flooding than that suggested by the visual reading method.

Figure 11 compares the features of the flooded areas between the visual reading and the $3 \mathrm{D}$ simulation methods by extracting the contour outlines based on the calculated results. Because a few areas were blocked by the lower heights of the banks around the rice fields, it was understood that the flowing waters with higher energies went slightly farther than the areas indicated by the visual reading method. This was particularly true in the A5 area, where the flooding damage areas identified by the 3D numerical analysis were much larger than those found by the visual reading method. This was confirmed when we visited the nearby town and asked the villagers about the affected areas, who explained that the damaged areas were greater than what was indicated by the visual reading. Therefore, the 3D simulation results reflecting flowing water energies calculated the damaged areas more accurately than the visual reading method.

Because this reservoir selected for the research is much smaller than the others managed by the KRC in South Korea, and many of the aged reservoirs are over 50 years old, the possible damage areas associated with these reservoirs contain higher safety risks and should be accurately determined through a 3D numerical simulation.

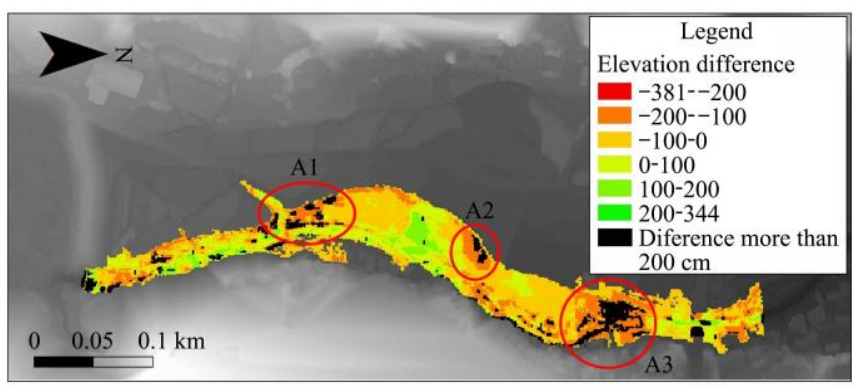

Figure 10 Differences in elevation between visual reading and 3D computer simulation

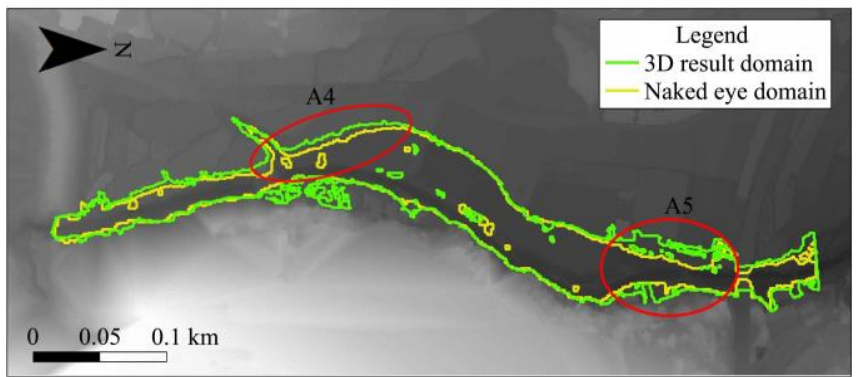

Figure 11 Comparison of flooded areas identified by visual reading and $3 \mathrm{D}$ simulation methods

\section{Conclusions}

A UAV was used to monitor the wide extent of area damaged by the 2014 collapse of the Goeyeon reservoir located in the Goeyeon-dong district in South Korea. The images obtained from the drone were used to produce accurate digital topographic maps using point cloud data. The flooded areas were analyzed using the finite volume method with the prepared DSM as the domain data. The following conclusions were obtained by comparing these simulation results with visual readings.

The DSM based on the UAV images indicated that the surface elevation information was faulty owing to natural or artificial obstacles such as trees, stream weirs, bridges, and a curved topologic geometry. If this error occurs at a point where the stream is flowing, it will be interpolated as higher than the river's actual elevation, which will change the direction and amount of stream flow. The most accurate method for calibrating the elevation is to use field survey information at the sites, although this is expensive. Instead, Kriging interpolation was applied as the spatial information algorithm, which topologically calibrates noncontinuous areas through a Gaussian process. Nevertheless, there were differences in the water depths between the 3D 
simulation and the visual reading methods. In the case of a 3D simulation analysis, the water level was higher than the visual reading method when there were obstacles, such as concrete weirs in the stream or when the stream was curved. In the simulation, flow patterns similar to a laminar flow were mainly found in areas with relatively low or high immersion depths, and the differences between visual readings were relatively reduced. However, the differences in the middle areas of the water depth could further increase because turbulence flows were found in the areas. This was confirmed when the local eyewitnesses were asked about the flood-damaged areas, who reported that the damaged areas were larger than what was estimated through a visual reading and more closely resembled the 3D simulation results. Thus, the 3D simulation results precisely reflected the energies of the flowing water because they calculated the hydrodynamic potential energies based on numerical simulation methods.

The rural areas in South Korea have thousands of widely distributed reservoirs for providing agricultural water. These are connected to narrow, densely populated streams. In the areas where the water flow is obstructed by weirs or natural terrain, it is likely that floods will occur owing to reverse flow dynamic energies. The current 1D or 2D models developed thus far cannot produce accurate results because the models reflect the subjective criteria of engineers, and the quality of the digital maps used for the simulations is low. In particular, they contain large errors in the calculated dynamic energy of a fluid by replacing such energy with a similar pseudo energy. In addition, the resolutions (1/1000 or 1/5000) of the numerical maps supplied by the National Geographic Information Institute in South Korea are too small to conduct accurate simulations. Therefore, existing models are not useful, despite their short simulation time. In one- and two-dimensional analyses, the slope, area, and flow resistance of the basin should be used as basic input data for empirical formulas such as the Kirpich equation. Because of this methodology, the entire targeted site is represented by a single average value. However, because a 3D dynamic simulation on a 3D terrain was conducted in this study, the characteristic values on each surface are accurately reflected. Therefore, it is possible for a 3D simulation to estimate the reach of a flood wave, flooded area, and other aspects. Additionally, this study accurately predicts the downstream areas flooded by a reservoir collapse through a 3D numerical fluid dynamic analysis based on high-resolution digital topographic maps produced through UAV imagery. In this paper, optimization conditions were suggested to conduct a complex $3 \mathrm{D}$ simulation based on a high-resolution digital map. A 3D numerical analysis has the potential to accurately assess these risk factors as well as the probable disaster effects of heavy rains and typhoons before any damage occurs.

Governments that manage disaster safety should carry out preliminary simulations to prepare for abnormal weather conditions such as heavy rains. This can be helpful to draw up a plan to secure the property and safety of the citizens through prior maintenance or evacuation plans in the case of actual heavy rains.

\section{Acknowledgements}

This work was supported by Creative-Pioneering Researchers Program through Seoul National University (SNU) in 2018-2020.

\section{[References]}

[1] Park J S, Jeon J B, Yoon S S. Evaluation of systematic for a small reservoir group based on system reliability technique. Journal of the
Korean Society of Agricultural Engineers, 2015; 57(3): 101-108. (in Korean)

[2] Lee D Y, Kim W T, Yoo C S. Climate change impacts on metrological drought and flood. Journal of Korea Water Resources Association, 2004; 37(4): 315-328. (in Korean)

[3] Park J Y, Joh H K, Jung I K, Jung K S, Lee J H, Kang B S, et al. Modeling downstream flood damage prediction followed by dam-break of small agricultural reservoir. Journal of the Korean Society of Agricultural Engineers, 2010; 52(6): 63-73. (in Korean)

[4] Choi H S. Study Trend on flood analysis by reservoir failure. Korean National Committee on Irrigation and Drainage (KCID), 2009; 16(1): 30-33. (in Korean)

[5] National Disaster Management Research Institute (NDMI). A report on the collapse of the bank of Sandae reservoir in Ahngang Province, Gyeongju-Si, Gyeongsangbuk-Do, South Korea, 2013.

[6] Kim J Y, Kim T H, Kim Y S, Kim J H. Stability analysis of embankment overtopping by initial fluctuating water level. Journal of The Korean Geotechnical Society, 2015; 31(8): 51-62. (in Korean)

[7] National Emergency Management (NEM). Study on Establishment Criteria of Dam Emergency Action Plan, South Korea, 2004.

[8] Kim S G, Song I H, Kim C. Comparison of flooding area estimation using gis and hydraulic model. Journal of the Korean Association of Geographic Information Studies, 2006; 9(3): 183-192. (in Korean)

[9] Cho W H, Han K Y, Ahn K H. Flood Risk mapping with FLUMEN model application. Journal of the Korean Society of Civil Engineers, 2010; 30(2): 169-177. (in Korean)

[10] Goodell C, Warren C. Flood inundation mapping using HEC-RAS Obras y Proyectos, 2006; 2: 18-23.

[11] Khattak M S, Anwar F, Saeed T U, Sharif M, Sheraz K, Ahmed A. Floodplain mapping using HEC-RAS and ArcGIS: A case study of Kabul River. Arabian Journal for Science and Engineering, 2016; 41(4): 1375-1390.

[12] Patel D P, Ramirez J A, Srivastava P K, Bray M, Han D. Assessment of flood inundation mapping of Surat city by coupled $1 \mathrm{D} / 2 \mathrm{D}$ hydrodynamic modeling: A case application of the new HEC-RAS 5. Natural Hazards, 2017; 89(1): 93-130.

[13] Tate E C, Maidment D R, Olivera F, Anderson D J. Creating a terrain model for floodplain mapping. Journal of Hydrologic Engineering, 2002; 7(2): 100-108.

[14] U.S. Army Corps of Engineers (USACE). Hec-Ras River Analysis System User's Manual 4.1, 2010.

[15] Alho P, Aaltonen J. Comparing a 1D hydraulic model with a 2D hydraulic model for the simulation of extreme glacial outburst floods. Hydrological Processes: An International Journal, 2008; 22(10): 1537-1547.

[16] Tayefi V, Lane S N, Hardy R J, Yu D. A comparison of one - and two - dimensional approaches to modelling flood inundation over complex upland floodplains. Hydrological Processes: An International Journal, 2007; 21(23): 3190-3202.

[17] Vasquez J A, Roncal J J. Testing River2D and FLOW-3D for sudden dam-break flow simulations. Proc., CDA 2009 Annual Conf., 3-8 October, Whistler, BC, Canada, 2009; pp.44-55.

[18] Choi W, Jeon J, Park J, Lee J, Yoon S. System reliability analysis of downstream spillways based on collapse of upstream spillways. Int $\mathrm{J}$ Agric \& Biol Eng, 2015; 8(4): 140-150.

[19] Hervouet J M, Petitjean A. Malpasset dam-break revisited with two-dimensional computations. Journal of Hydraulic Research, 1999; 37(6): 777-788.

[20] Valiani A, Caleffi V, Zanni A. case study: Malpasset dam-break simulation using a two-dimensional finite volume method. Journal of Hydraulic Engineering, 2002; 128(5): 460-472.

[21] Begnudelli L, Sanders B F. Simulation of the St. Francis dam-break flood. Journal of Hydraulic Research, 2007; 133(11): 1200-1212.

[22] Park J C, Jung I W, Chang H J, Kim M K. Optimization of PRISM parameters and digital elevation model resolution for estimating the spatial distribution of precipitation in South Korea. Journal of the Korean Association of Geographical Information Studies, 2012; 15(3): 36-51. (in Korean)

[23] Kim J M, Park S S, Kim J H, Ahn S W, Park S Y, Kim Y S. Accuracy evaluation and terrain model automation of reservoir using unmanned aerial vehicle system. Journal of the Korean Society of Agricultural Engineers, 2017; 59(2): 57-67. 
[24] Everaerts J. The use of unmanned aerial vehicles (UAVS) for remote sensing and mapping. The International Archives of the Photogrammetry, Remote Sensing and Spatial Information Sciences, 2008; 37: 1187-1192.

[25] Lin Z J. UAV for mapping-low altitude photogrammetric survey. International Archives of Photogrammetry and Remote Sensing, 2008; 37 : 1183-1186.

[26] Park J K, Park J H. Reservoir failure monitoring and identified by the UAV aerial images. Korean Review of Crisis and Emergency Management, 2015; 11(4): 155-167.

[27] Kim D I, Song Y S, Kim G H, Kim C W. A study on the application of UAV for Korean land monitoring. Journal of the Korean Society of Surveying. Geodesy, Photogrammetry and Cartography, 2014; 32(1): 29-38. (in Korean)

[28] Zhang C, Kovacs J M. The application of small unmanned aerial systems for precision agriculture: A review. Precision Agriculture, 2012; 13(6): 693-712.

[29] Park J K, Park J H. Applicability evaluation of agricultural subsidies inspection using unmanned aerial vehicle. Journal of the Korean Society of Agricultural Engineers, 2016; 58(5): 29-37.

[30] Lee D, Choi J, Shin S, Yi J. A study on proper number of subbasin division for runoff analysis using Clark and ModClark method in Midsize Basins. Journal of the Korean Society of Civil Engineers,. 2013; 33(1): 157-170. ,

[31] Savage B M, Johnson M C. Flow over ogee spillway: Physical and numerical model case study. J Hydraulic Eng., 2001; 127(8): 640-649. ,

[32] Johnson M C, Savage B M. Physical and numerical comparison of flow over ogee spillway in the presence of tailwater. J Hydraulic Eng., 2006; 132(12): 1353-1357.
[33] Chanel P G, Doering J C. Assessment of spillway modeling using computational fluid dynamics. Can J Civil Eng., 2008; 35(12): 1481-1485.

[34] Kocaman S, Hatice O C. The effect of lateral channel contraction on dam break flows: Laboratory experiment. J Hydrol, 2012; 432/433: 145-153.

[35] Saad Y, Schultz M H. GMRES: A generalized minimal residual algorithm for solving nonsymmetric linear systems. SIAM J Sci Stat Comput, 1986; 7(3): 856-869.

[36] Ashby S F, Manteuffel T A, Saylor P E. A Taxonomy for conjugate gradient methods. SIAM J Numer Anal, 1990; 27: 1542-1568.

[37] Barrett R, Berry M, Chan T F, Demmel J, Donato J M, Dongarra J, et al. Templates for the solution of linear systems: Building blocks for iterative methods. In Philadelphia, SIAM, 1994; 104p. doi:http://dx.doi.org/10.1137/1.9781611971538.

[38] Anderson S. An evaluation of spatial interpolation methods on air temperature in Phoenix, AZ. Department of Geography, Arizona State University, 2002; 0104.

[39] Cressie N. Statistics for spatial data. New York: John Wiley \& Sons, Inc. $2015 ; 928 p$.

[40] Jeon J B, Na S I, Yoon S S, Park J H. Spatial distribution of $\mathrm{CO}_{2}$ absorption derived from land-cover and stock maps for Jecheon, Chungbuk Province. Journal of Korean Society of Rural Planning, 2013; 19(2): 121-128. (in Korean)

[41] Di Piazza A, Conti F L, Noto L V, Viola F, La Loggia G. Comparative analysis of different techniques for spatial interpolation of rainfall data to create a serially complete monthly time series of precipitation for Sicily, Italy. International Journal of Applied Earth Observation and Geoinformation, 2011; 13(3): 396-408. 